\title{
Adaptação transcultural do Myocardial Infarction Dimensional Assessment Scale (MIDAS) para a língua portuguesa brasileira
}

\author{
Cross-cultural adaptation of the Myocardial Infarction Dimensional \\ Assessment Scale (MIDAS) to the Brazilian Portuguese language
}

Bruno Henrique Fiorin ${ }^{1}$

Elizabete Regina Araújo de Oliveira ${ }^{1}$

Rita Simone Lopes Moreira ${ }^{2}$

Bráulio Luna Filho ${ }^{2}$

${ }^{1}$ Departamento de

\begin{abstract}
From the evaluation of the factors that affect quality of life (QOL) it is possible to plan interventions that lead to the improved well-being of patients. The scope of this study was to conduct the cross-cultural adaptation of the Myocardial Infarction Dimensional Assessment Scale (MIDAS) questionnaire to the Portuguese language, seeking the necessary semantic, idiomatic, conceptual and cultural equivalence. The theoretical framework of Guillemin, Bombardier and Beaton was used, fulfilling the following steps: translation, back translation, evaluation of the authors, peer review and pre-testing. After all the tests, the semantic, idiomatic, conceptual and cultural equivalence was achieved. The scale proved to be easy to use and was clinically important. MIDAS was validated in terms of its semantic, idiomatic, conceptual and cultural equivalences. Subsequently, the measurement equivalence will be evaluated to verify the psychometric properties.
\end{abstract}

Key words Quality of life, Validation study, Questionnaire
Resumo A partir da avaliação dos fatores que incidem na qualidade de vida (QV) é possível planejar as intervenções que proporcionam melhoria do bem-estar dos pacientes. Realizar a adaptação transcultural do questionário Miocardial Infarction Dimensional Assessment Scale (MIDAS) para lingua portuguesa, buscando as equivalências semântica, idiomática, conceitual e cultural. Foi utilizado o referencial teórico de Guillemin, Bombardier e Beaton, cumprindo as seguintes etapas: tradução, retrotradução, avaliação dos autores, banca de juizes e pré-teste. Após todas as avaliações, foram alcançadas as equivalências semântica, conceitual, idiomática e cultural. A escala mostrou-se de fácil aplicação e importância clínica. O MIDAS encontra-se validado no que se refere às equivalências semânticas, idiomáticas, conceituais e culturais. Posteriormente, será avaliada a equivalência de mensuração, para verificar as propriedades psicométricas.

Palavras-chave Qualidade de vida, Estudo de validação, Questionário 


\section{Introdução}

A Organização Mundial da Saúde (OMS) define que qualidade de vida $(\mathrm{QV})$ é a percepção do indivíduo sobre sua posição na vida, considerada no contexto de cultura, de valores nos quais vive, e elabora seus objetivos, expectativas, padrões e preocupações $^{1}$. A QV é um tema de grande importância para a saúde, por envolver aspectos inerentes ao indivíduo e considerar a percepção da pessoa acerca do seu estado de saúde em diferentes dimensões da vida. Dessa forma, é necessária uma avaliação mais pontual acerca do estado de saúde das pessoas, inserindo a qualidade de vida como indicador de eficácia e avaliação de intervenções em saúde ${ }^{1-3}$.

A melhoria da qualidade e do estilo de vida passou a ser um dos desfechos relevantes tanto nas práticas assistenciais quanto nas políticas públicas para a promoção de saúde e prevenção de doenças ${ }^{4}$. A partir da avaliação dos mecanismos que incidem na qualidade de vida é possível o planejamento de intervenções ou tratamentos que possam proporcionar a melhoria do bem-estar dos pacientes.

Os instrumentos que avaliam a qualidade de vida em paciente infartado possuem o objetivo de mensurar o efeito da doença cardiorrespiratória, quantificando aspectos como disfunções e desconfortos físicos-emocionais, contribuindo no planejamento dos tratamentos e o sucesso terapêutico ${ }^{6,7}$.

A avaliação objetiva da QV é realizada por meio de questionários ou formulários. Esses questionários dividem-se em genéricos e específicos. Os genéricos possibilitam a mensuração de diversos domínios como capacidade funcional, aspectos físicos, dor, estado geral de saúde, vitalidade, aspectos sociais, emocionais e saúde mental $^{8}$. Já os instrumentos específicos, apesar de também avaliarem diversos fatores, têm como foco principal aqueles limitantes e as características particulares que a doença em questão ocasiona nos pacientes ${ }^{7,8}$.

Vários estudos propõem a realização da adaptação transcultural de instrumentos de pesquisa, apesar de não haver um consenso de qual substrato teórico e metodológico utilizar como guia. Nesse sentido, modelos explorados por diferentes autores são recomendados para a execução desse processo $^{1,9-11}$.

O instrumento uma vez adaptado estará então apto para ser utilizado em uma população específica. Porém, deve ser apreciado por profissionais interessados, já que o aperfeiçoamento do instrumento adaptado depende de discussões e críticas contínuas entre os profissionais e a população participante, para se alcançar o escopo de eficácia, ou seja, para que apresente sensibilidade e especificidade sobre o objeto avaliado ${ }^{11-13}$.

Em revisão sistemática com meta-análise, cujo objetivo era identificar instrumentos traduzidos para o português para mensurar a QV em pacientes com síndrome coronariana aguda, os seguintes questionários de qualidade de vida foram mais utilizados: SF-36, Mac New, WHOQOL, Seattle, IPQ e NHP. Desses, somente o Mac New é especifico para pacientes infartados ${ }^{14}$. Este último faz uma síntese dos estudos de questionários sobre avaliação de QV, traduzidos para o português, apresenta falhas nos métodos de validação, denotando que informações sobre a evolução da qualidade de vida em pacientes brasileiros ou portugueses com evento coronariano agudo ainda são necessárias.

Ressalte-se que a versão original do QLMI Mac New foi projetada para avaliar a eficácia de um programa de reabilitação para pacientes após terem problemas cardíacos ${ }^{15,16}$. Essa característica não é comum no Brasil, pois não possuímos programas de reabilitação que consigam dar cobertura a essa população $0^{17,18}$.

Diante desse cenário, propõem-se a adaptação transcultural MIDAS para língua portuguesa brasileira, originariamente criado no Reino Unido para preencher as lacunas dos outros instrumentos $^{19}$. É um questionário, autoadministrado, que abrange sete dimensões relacionadas com o estado de saúde, quais sejam: atividade física, insegurança, reação emocional, dependência, dieta, medicamentos e preocupações com efeitos colaterais.

\section{Metodologia}

Trata-se de estudo metodológico de abordagem quantitativa com o intuito de adaptar o instrumento especifico de qualidade de vida - o Myocardial Infarction Dimensional Assessment Scale (MIDAS) - para ser aplicado em nosso meio.

Na realização da tradução e adaptação transcultural do instrumento, seguiu-se as orientações propostas por Guillemin et al. ${ }^{1}$. Após obtida a autorização dos autores, o instrumento foi encaminhado para dois tradutores. Ambos possuíam proficiência em língua inglesa e desenvolviam trabalhos de versões de artigos e materiais científicos do inglês para o português e vice-versa, e estavam cientes dos objetivos do estudo. $\mathrm{O}$ tradu- 
tor 1 ( $\mathrm{t} 1$ ) foi considerado o tradutor especialista, por possuir experiência no manejo de textos na área da saúde, bem como em processo de tradução e docência de inglês instrumental. Porém, apesar de ser familiarizado com a temática, ainda não tivera contato com o questionário em questão. O outro tradutor (t2) foi considerado leigo, uma vez que faz traduções de maneira geral, porém não possuía conhecimento instrumental na língua inglesa no campo da saúde.

As versões traduzidas foram comparadas pelos autores. O índice de concordância entre as traduções foi de $90 \%$. Os itens traduzidos de forma diferente foram discutidos para alcance de um consenso entre os tradutores e pesquisadores, obtendo-se assim a versão inicial traduzida do MIDAS- T12.

A tradução reversa ou retrotradução para língua de origem foi realizada por outros dois tradutores, de maneira independente. Esses eram naturais de países de língua inglesa e haviam proficiência em língua portuguesa. Ambos também tinham vasta experiência em traduções e prestavam serviços para empresas de traduções. Vale destacar aqui que os tradutores receberam apenas a versão T12 e o mesmo processo de comparação dos dados foi realizado pelos pesquisadores e tradutores.

Essa é uma das etapas de validação do instrumento em que verifica se a versão obtida reflete o mesmo conteúdo da versão original, permitindo verificar as inconsistências e os erros conceituais cometidos durante as traduções. Assim, após a avaliação pelos pesquisadores, obteve-se uma versão unificada. Essa versão final foi submetida à análise dos autores originais do instrumento para a devida apreciação da conservação do sentido original do instrumento. Após essa avaliação, foram realizadas as alterações necessárias na versão T12 para preservação do sentido, obtendo-se assim a versão final do processo de tradução.

Após consolidar as versões e ter o parecer favorável dos autores originais do instrumento, essas foram avaliadas por um comitê de juízes, composto por cinco profissionais da área da saúde e com experiência na área de pesquisa. Esses juízes tinham fluência na língua inglesa e tiveram a incumbência de julgar e avaliar as equivalências semânticas, idiomáticas e as equivalências culturais e conceituais do instrumento.

A escolha da banca foi realizada de maneira aleatória, buscando-se na plataforma Lattes profissionais que, além da experiência de campo, também tinham desenvolvido trabalhos acadêmicos na área da cardiologia relacionados com pacientes infartados. Todos tinham no mínimo mestrado e mais de dois anos de atuação profissional. Além dos cinco especialistas, participaram do comitê de juízes os tradutores para auxiliar na discussão no processo de equivalências. Cada integrante da banca avaliou primeiramente o instrumento e suas traduções de maneira individual e após esta etapa foi realizado a discussão em grupo, com todos os juízes, para a adequação dos termos e avaliação das discrepâncias do processo de tradução.

Foram aceitos como equivalentes os itens que obtiveram $80 \%$ de concordância entre os juízes. Os itens com um índice de concordância menor seriam modificados de acordo com as sugestões propostas pelos juízes, no momento da discussão, juntamente com os pesquisadores. Para isso foi aplicado o Índice de Validade de Conteúdo (IVC) utilizado nos instrumentos de avaliação da equivalência semântica, conceitual, cultural e idiomática $^{20,21}$. Para avaliar a relevância e representatividade, as respostas foram categorizadas: 1 = não relevante ou não representativo, 2 = item necessitado de grande revisão para ser representativo, 3 = item necessitado de pequena revisão para ser representativo, $4=$ item relevante ou representativo. Para cada item do instrumento, os juízes deveriam comparar a sentença original com as traduções e atribuir uma nota de acordo com IVC.

O escore do índice foi calculado por meio da soma de concordância dos itens que foram marcados por " 3 " ou " 4 " pelos juízes. Os itens que receberam pontuação " 1 ” ou " 2 " deveriam ser revisados ou eliminados, baseando-se nas sugestões do comitê. Assim, o IVC foi definido como a proporção de itens que receberam uma pontuação de 3 ou 4 pelos juízes.

O pré-teste constituiu a última etapa do processo de tradução e adaptação transcultural. Nessa fase, a versão final do instrumento foi submetida a aplicação para análise da compreensibilidade dos itens ${ }^{9}$. O pré-teste foi realizado no ambulatório de cardiologia do HUCAM - Hospital Universitário Cândido Mendes, para participar desta etapa os pacientes deveriam ter histórico de IAM registrado em prontuário, ter mais de 18 anos e aceitar de maneira voluntária a participar do estudo.

Para analisar a compreensibilidade da escala traduzida e adaptada foi utilizada a técnica da prova, na qual, após a leitura de cada pergunta, o pesquisador arguiu o entrevistado para que esse explicasse o questionamento, buscando assim a validade de face do instrumento, ou seja, 
uma avaliação subjetiva por aqueles que utilizam a escala, a fim de verificar a consistência na mensuração do fenômeno que se avalia ${ }^{1}$.

A partir desses resultados, obteve-se a versão traduzida do MIDAS para sua versão em português brasileiro, a qual se encontra no Quadro 1.

Essa pesquisa foi aprovada pelo Comitê de Ética e Pesquisa da Universidade Federal do Espírito Santo. Os participantes da pesquisa assinaram o termo de consentimento livre e esclarecido - TCLE.

\section{Resultados}

O processo de adaptação transcultural teve início com a investigação da equivalência conceitual. Ao se comparar as duas traduções, houve concordância em mais de 90\% dos termos traduzidos. Os termos ou sentenças que apresentaram discordância ou traduções diferentes foram julgados pela equipe de unificação.

Os termos/sentenças que tiveram traduções diferentes entre o T1 e T2 foram discutidos entre os pesquisadores e o Quadro 2 - “Termos que foram discutidos pela equipe de unificação no processo de Tradução do Midas" - apresenta estas informações.

Em meio ao processo de consolidação da T1 e da T2, alguns aspectos foram reavaliados para uniformização do instrumento. As perguntas subsequentes do questionário começaram com letra minúscula, pois se entende que sejam continuidade da pergunta anterior: "Após seu ataque cardíaco com que frequência durante a última semana você..... Essa foi uma sugestão proposta pelo T1 e que os pesquisadores consideraram válida.

Ao traduzir o termo "Occasionally", T1 sugeriu "raramente" e T2 "ocasionalmente". Optou-se pelo último termo "raramente", considerando que a primeira sugestão é mais utilizada como sinônimo de "às vezes", "sometimes" em inglês.

Posteriormente, a versão T12 foi submetida à retrotradução para língua de origem. Esse processo foi realizado por dois tradutores de maneira independente, como já referido. Nessa etapa foram produzidas duas traduções, as versões TR1 e TR2.

Por solicitação dos autores, as duas traduções foram unificadas para que os mesmos pudessem realizar a concordância com a preservação do sentido e a manutenção do objetivo do instrumento. A equipe de unificação novamente realizou a integração das traduções (TR1 e a TR2), obtendo uma nova versão, a TR12, em língua inglesa. Como não houve discrepâncias entre as traduções, os itens não equivalentes foram apenas julgados pela equipe. A versão TR12 foi, portanto, encaminhada aos autores para avaliação. Estes, por sua vez, fizeram algumas sugestões de adequações na versão T12.

Um dos pontos destacados pelos autores foi a necessidade de frisar que o instrumento avalia o último ataque cardíaco, ou seja, as alterações no quadro de saúde após o último infarto.

Em relação à questão 4 (Felt slowed down?), os autores sugeriram detalhar que o sentido de “felt slowed down” está relacionado ao desânimo em realizar a atividade física, pois somente utilizar o termo "desanimado" poderia deixar o entrevistado em dúvida e não abranger toda significância necessária.

O termo "breathless", que foi consolidado como "sem fôlego", tem um sentido mais amplo, sendo mais bem caracterizado como "falta de ar intensa", uma vez que os autores entendem que o indivíduo pode apresentar perda do fôlego, mas o sintoma que caracteriza uma alteração na condição de qualidade de vida é a falta de ar intensa.

Outro termo discutido com os autores foi “irritable”, o qual literalmente está vinculado à irritação. Contudo, os mesmos afirmaram que o sentido a ser explorado é um processo emotivo, conjunto de emoções e não somente a relação com o sentimento de raiva. Desta forma a tradução sugerida para "felt irritable" foi " se sentiu emotivo".

Em relação ao termo "independence" na questão 27 (Felt that you have lost your independence?), apesar da tradução literal sugerir a avaliação da perda de independência do paciente, os autores destacam, que o intuito desta pergunta é avaliar se ele está mais dependente dos outros, sugerindo a seguinte tradução: "Sentiu que você depende de outras pessoas?"

Todas essas considerações foram acatadas e as modificações implementadas na versão T12, que foi, então, submetida à banca de juízes.

A banca de juízes foi constituída por um grupo multiprofissional, formado por cinco integrantes, sendo um médico, dois enfermeiros, um psicólogo e um fisioterapeuta, além dos tradutores envolvidos no estudo. Todos os integrantes eram profissionais atuantes na área da cardiologia, tendo experiência no manejo e acompanhamento do paciente após infarto.

Para cada um deles foi enviado o questionário na versão original, a primeira versão traduzida, a versão modificada após avaliação dos autores e também o instrumento para determinação 
Quadro 1. Escala de Avaliação Multidimensional Pós-Infarto Agudo do Miocárdio (MIDAS-35).

\begin{tabular}{l} 
Por favor, leia antes de completar este questionário: \\
\hline O objetivo do questionário é fazer perguntas sobre sua saúde e como você se sente depois de ter sofrido o último ataque \\
cardíaco. Se você teve mais de um ataque cardíaco, favor responder às perguntas considerando o ataque cardíaco mais recente. \\
Se você não tem certeza de como responder quaisquer das questões, tente pensar sobre sua saúde após o ataque cardíaco e \\
como ela foi afetada. Se você não sabe ao certo como descrever seus sintomas, registre quaisquer sintomas que você possa ter \\
tido.
\end{tabular}

Não gaste muito tempo pensando sobre suas respostas, provavelmente sua resposta mais imediata tende a ser a mais precisa.

Após seu ataque cardíaco, com que frequência durante $a$ última semana você...

1. pensou duas vezes antes de iniciar uma atividade física (por exemplo, tarefas domésticas ou sair para fazer compras)?

Por favor, assinale apenas um círculo para cada questão.

2. teve sintomas de angina (por exemplo, dor ou aperto no peito)?

3. teve angina (dor no peito ou sensação de aperto) que afetou sua vida?

4. sentiu-se desanimado para realizar atividades diárias?

5. sentiu-se sem força?

6. sentiu-se com falta de ar intensa?

7. percebeu que a mudança de temperatura fez com que sua dor piorasse?

8. sentiu-se frustrado com suas limitações?

9. sentiu necessidade de descansar?

10. sentiu que reduziu seu ritmo de vida social?

11. sentiu que não poderia realizar as tarefas domésticas?

12. percebeu que a temperatura ambiental fez a sua dor pior?

13. sentiu medo de ter outro ataque do coração?

14. sentiu-se isolado?

15. sentiu-se sozinho?

16. sentiu-se ansioso em viajar?

17. sentiu-se vulnerável?

18. sentiu-se inseguro?

19. sentiu que sua confiança foi afetada?

20. sentiu-se ansioso em relação à morte?

21. preocupou-se ou sentiu-se ansioso com o futuro?

22. sentiu-se emotivo?

23. sentiu-se deprimido?

24. sentiu-se mal humorado?

25. sentiu-se estressado?

26. percebeu que sua família ou seus amigos estão lhe superprotegendo?

27. sentiu que você depende de outras pessoas?

28. sentiu que precisava mais das outras pessoas?

29. sentiu-se preocupado com a sua dieta?

30. sentiu-se preocupado com o seu nível de colesterol?

31. preocupou-se com o seu peso?

32. preocupou-se em tomar remédio?

33. preocupou-se com os efeitos colaterais de seus remédios?

34. sentiu mais frio?

35. experimentou efeitos colaterais (por exemplo, mãos ou pés frios / ir ao banheiro à noite) de sua medicação?

\section{\begin{tabular}{l|l|l|l|l|} 
Nunca & Raramente & Às vezes & Frequentemente & Sempre
\end{tabular}}
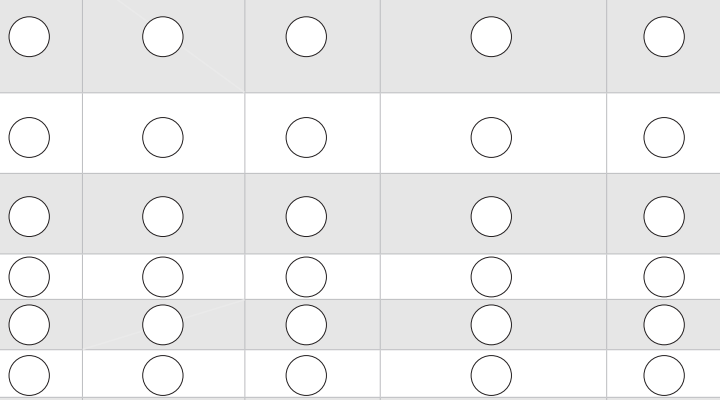

0

$0 \quad 0$

\section{0}

0

0

$\bigcirc$

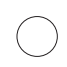

0

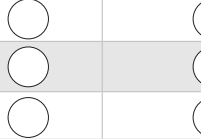

$\bigcirc$
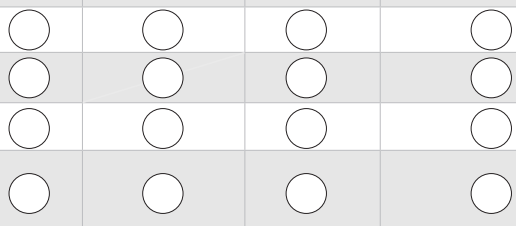

(
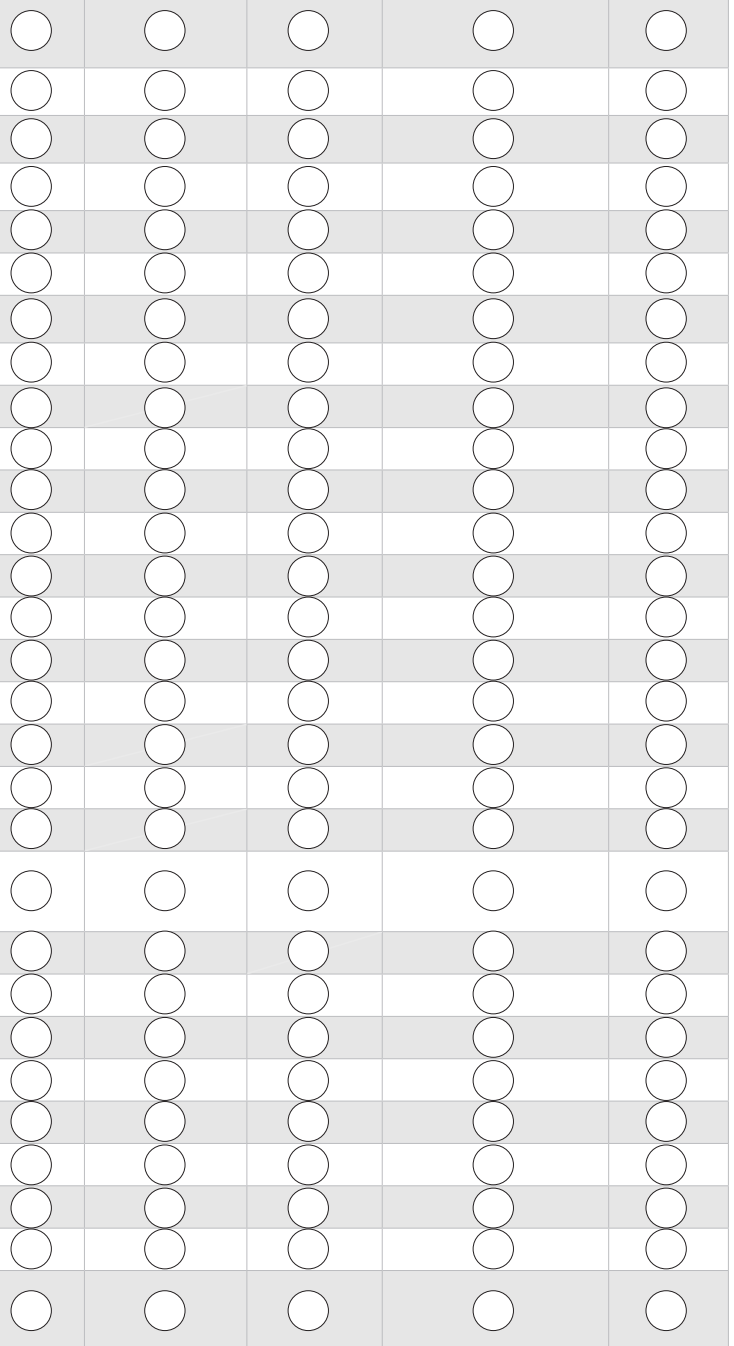
Quadro 2. Termos que foram discutidos pela equipe de unificação no processo de Tradução do Midas.

\begin{tabular}{|l|l|l|l|}
\hline \multicolumn{1}{|c|}{$\begin{array}{c}\text { Termo original } \\
\text { (MIDAS) }\end{array}$} & Tradução pelo tradutor $\mathbf{1}$ & Tradução pelo tradutor $\mathbf{2}$ & \multicolumn{1}{|c|}{$\begin{array}{l}\text { Termo escolhido pela } \\
\text { equipe de unificação }\end{array}$} \\
\hline Dimensional & Dimensional & Multidimensional & Multidimensional \\
\hline How often & Quantas vezes & Com que frequência & Com que frequência \\
\hline Housework & Tarefas domésticas & Limpar a casa & Tarefas domésticas \\
\hline Felts lowed down & Sentiu-se devagar & $\begin{array}{l}\text { Sentiu-se fazendo suas } \\
\text { atividades de maneira lenta }\end{array}$ & Sentiu-se desanimado \\
\hline $\begin{array}{l}\text { Felt you have a reduced } \\
\text { social life }\end{array}$ & $\begin{array}{l}\text { Sentiu que você tem uma } \\
\text { vida social reduzida }\end{array}$ & $\begin{array}{l}\text { Diminui seu ritmo de vida } \\
\text { social }\end{array}$ & $\begin{array}{l}\text { Sentiu que diminui seu } \\
\text { ritmo de vida social }\end{array}$ \\
\hline $\begin{array}{l}\text { Felt anxious about } \\
\text { travelling }\end{array}$ & $\begin{array}{l}\text { Sentiu-se ansioso em viajar } \\
\text { Sentiu ansiedade com } \\
\text { relação a qualquer } \\
\text { probabilidade de viajar }\end{array}$ & $\begin{array}{l}\text { Sentiu-se ansioso em } \\
\text { viajar }\end{array}$ \\
\hline $\begin{array}{l}\text { Felt anxious about } \\
\text { dying }\end{array}$ & $\begin{array}{l}\text { Sentiu-se ansioso sobre } \\
\text { morrer }\end{array}$ & $\begin{array}{l}\text { Teve pensamentos sobre } \\
\text { morte }\end{array}$ & $\begin{array}{l}\text { Sentiu-se ansioso em } \\
\text { relação à morte }\end{array}$ \\
\hline $\begin{array}{l}\text { Felt your family } \\
\text { or friends are over } \\
\text { protective }\end{array}$ & $\begin{array}{l}\text { Sentiu que sua família } \\
\text { ou amigos estão mais } \\
\text { proteção }\end{array}$ & $\begin{array}{l}\text { Achou que sua família } \\
\text { e seus amigos se } \\
\text { preocupavam demais }\end{array}$ & $\begin{array}{l}\text { Percebeu que sua família } \\
\text { ou amigos estão lhe } \\
\text { superprotegendo }\end{array}$ \\
\hline $\begin{array}{l}\text { Felt you have to rely on } \\
\text { others }\end{array}$ & $\begin{array}{l}\text { Sentiu que você precisa } \\
\text { confiar em outras pessoas }\end{array}$ & $\begin{array}{l}\text { Sentiu-se dependentes dos } \\
\text { outros }\end{array}$ & $\begin{array}{l}\text { Sentiu que você tem que } \\
\text { confiar em outras pessoas }\end{array}$ \\
\hline Tablets & Comprimidos & Remédio & Remédio \\
\hline Experienced & Experimentou & Sentiu & Experimentou \\
\hline
\end{tabular}

Fonte: Dados de pesquisa (2013).

do índice de validade de conteúdo (IVC) semântico, idiomática, conceitual e cultural. Esses instrumentos quantificaram a impressão dos juízes acerca da adaptação do MIDAS $^{20,21}$.

A maioria dos itens do instrumento foi considerada equivalente, uma vez que o grau de concordância entre os juízes foi superior a $80 \%$, ou seja, a pontuação em relação ao IVC variou de 3 e 4 .

Os conceitos de equivalências semântica, idiomática, cultural e conceitual foram trabalhados baseando-se nos direcionamentos propostos pelos autores e explorados pelos juízes ${ }^{11,13}$.

Em relação ao item da questão cinco "felt with out energy", que havia sido traduzido como "se sentiu sem energia", foi sugerido pelos juízes que esse fosse substituído por "sem força", por ser culturalmente mais adequado. Após esta discussão a tradução foi modificada para "se sentiu sem força".

Na questão sete, "had chest pain or tightness when dopings physical activity", cuja tradução era "teve dor no peito ou sensação de aperto ao fazer atividade física", foi sugerida a modificação "teve dor no peito ou sensação de aperto ao fazer suas atividades diárias". Esta adequação esta rela- cionada ao desencadeamento de sintomas devido ao esforço físico em relação as atividades de vida diária.

No item 12, em que se questiona "perceived that the ambient temperature caused your pain to worsen", a tradução era "percebeu que a temperatura ambiental fez a sua dor pior", foi sugerido que tentasse melhor o entendimento de como a temperatura ambiental pode afetar a dor. Dessa forma, o item foi modificado para "percebeu que a mudança de temperatura fez com que sua dor piorasse"

No item 15 "felt isolated" foi apresentada a ideia de que o isolamento é um estado muito complexo, assim, como o instrumento aborda questões cotidianas, seria interessante a substituição por um termo mais comum que pudesse expressar uma situação que anteceda o isolamento. Assim esse termo substituído por "sozinho".

No item 21 "was concerned or felt anchos or worried about the future", a tradução era "se preocupou ou sentiu ansiedade ou preocupado sobre o futuro", mas os juízes julgaram que a repetição de palavras trazia confusão ao paciente. Dessa maneira houve o seguinte rearranjo: "se preocupou ou sentiu-se ansioso com o futuro". 
O item 22 também foi questionado, pois quatro dos cinco juízes não concordaram com a versão original "felt irritable" ter sido traduzida como "sentiu-se emotivo". Mas, como foi uma sugestão dos autores originais do questionário, essa modificação de tradução não foi feita, uma vez que a primeira preserva o sentido do instrumento original e caracteriza um termo que possui equivalência na língua portuguesa brasileira.

$\mathrm{Na}$ questão 28 "felt you have to depend anothers", a tradução traz o termo confiança, porém 3 membros do comitê julgaram que confiança é algo muito complexo com muito envolvimento entre as pessoas. A tradução deveria então expressar mais o sentido de dependência. Assim, a tradução foi "sentiu que precisava mais das outras pessoas".

Após a realização da avaliação da banca de juízes, o instrumento foi considerado satisfatório em relação às equivalências apresentadas e foi submetido ao pré-teste, esta etapa foi realizada no Hospital Universitário Cassiano de MoraisHUCAM, no ambulatório de cardiologia.

No total, participaram dessa etapa do estudo 20 pacientes, sendo 14 do sexo feminino e 6 do sexo masculino. A idade desses variou de 42 a 89 anos e o nível de escolaridade predominante apresentava menos de 5 anos do Ensino Fundamental. Vale destacar que nenhum dos sujeitos da amostra possuía curso superior. Além do IAM, onze pacientes também eram hipertensos, dois possuíam diabetes, um possuía valvulopatia, um aterosclerose e outro insuficiência cardíaca.

Para essa etapa do processo foi utilizada a técnica de sondagem, cujo objetivo era, após cada questão, solicitar ao paciente que esse explicasse o que havia entendido da pergunta. Além disso, ao terminar todo o questionário, questionavase ao paciente se houve alguma dificuldade para responder o questionário. Caso houvesse alguma dúvida era questionado ao paciente em qual palavra ou sentença e se ele sugeria alguma mudança nas perguntas.

Nesse processo nenhum dos participantes fez qualquer questionamento acerca das questões. Portanto, após cada pergunta, todos os participantes conseguiram sintetizar de maneira satisfatória o sentido de cada item apresentado. O tempo médio para responder o questionário foi de $19,15 \mathrm{~min}$.

Cada uma das 35 perguntas que compõem o MIDAS é marcada na mesma direção, com a pontuação aumentando à medida que o estado de saúde relatado se torna pior. Assim, todas as questões dispõem de cinco possibilidades de resposta que denotam desde a ausência de sintomas, caracterizado pela opção "nunca" (pontuando 0), até as que representam maior gravidade, pontuando de 1 a 4 nas opções de resposta para cada item ${ }^{19}$.

Como a função do MIDAS é indicar a extensão dos problemas de saúde em cada domínio avaliado, cada subescala é transformada para ter um intervalo de 0 (o melhor estado de saúde como medida no questionário) até 100 (o pior estado de saúde como medida no questionário). Nesse ponto vale destacar que cada subescala é calculada como se segue: a pontuação de cada subclasse ou domínio é o total de escores brutos de cada item da escala dividido pelo escore bruto máximo possível, que é 4, multiplicado por 100 . E a pontuação total do instrumento pode ser calculada pela soma dos sete domínios, dividindo o somatório por 7 .

Os autores encaminharam algumas orientações gerais do instrumento: caso o paciente não respondesse alguma pergunta, deveria ser considerada a média atribuída ao domínio ao qual aquela pergunta fazia parte para aquela questão. Caso houvesse mais que uma resposta para a mesma pergunta, deveria ser considerada a que representasse o pior estado de qualidade de vida.

\section{Discussão}

O processo de adaptação cultural busca as equivalências necessárias para a aplicabilidade e reprodutibilidade do questionário em nacionalidades diferentes. Dessa forma, a avaliação da equivalência conceitual e cultural consiste na exploração do construto de interesse e dos pesos dados aos diferentes domínios constituintes no local (país, região, cidade) de origem e na população-alvo em que o questionário será aplicado.

Nesse sentido, a equivalência cultural referese às situações evocadas ou retratadas nos itens que correspondem às vivências diárias. Por outro lado, a equivalência conceitual representa a coerência em relação àquilo que se propõe medir ${ }^{1,9,11}$.

Já a avaliação da equivalência semântica e idiomática envolve a capacidade de transferência de sentido dos conceitos contidos no questionário original para a versão traduzida, propiciando um efeito nos respondentes semelhante nas duas culturas. Nesse sentido, a equivalência semântica diz respeito à correspondência no significado das palavras, enquanto a equivalência idiomática se refere ao uso de expressões que sejam comuns em ambos os países ou que retratem situações compatíveis. 
Vale destacar que alguns itens sofreram mudança após a avaliação dos juízes para proporcionar melhor equivalência, pois não alcançaram a concordância de $80 \%$, como já explicado.

Após a realização da avaliação da banca de juízes, o instrumento foi considerado satisfatório em relação às equivalências. As alterações solicitadas foram executadas de maneira a garantir a melhor sensibilidade do questionário.

De acordo com os autores ${ }^{1}$, um instrumento adaptado é aquele que pode ser compreendido por uma criança de 12 anos. Neste estudo a amostra do pré-teste foi formada de maneira heterogênea, por sujeitos de baixa escolaridade. Nesta condição o MIDAS se mostrou eficaz, no que se refere a adaptação transcultural, não apresentando qualquer questionamento acerca das questões por parte dos entrevistados ${ }^{22}$.

Finalizando, além das equivalências afirmadas pelos juízes, o MIDAS apresentou validade de face, uma vez que foi de fácil aplicação na população alvo, não havendo dúvida em relação ao sentido dos termos e dificuldades para responder cada item. Assim sendo, o MIDAS encontra-se adaptado transculturalmente para língua portu- guesa brasileira. Porém, vale ressaltar que, para sua maior eficácia e acurácia, faz-se necessária a avaliação das propriedades psicométricas, a qual será realizada em estudo posterior.

\section{Conclusões}

Após as etapas realizadas e os processos metodológicos executados, pode-se afirmar que o MIDAS está adaptado culturalmente para ser aplicado na população de língua portuguesa brasileira, no que se refere as equivalências semântica, conceitual, idiomática e cultural do processo de adaptação transcultural.

O MIDAS demonstrou ser um instrumento de fácil aplicação, útil para clínica e de bom entendimento pela população. Tem a abrangência de vários domínios que perpassam o constructo da QV do paciente após o IAM.

Apesar de adaptado nas etapas avaliadas, fazse necessário, para melhor aplicabilidade do instrumento, a realização da verificação das propriedades psicométricas, que será tema de próximo estudo.

\section{Colaboradores}

BH Fiorin, ERA Oliveira, RSL Moreira e B Luna Filho participaram do trabalho e revisaram e concordaram com o conteúdo do artigo. 


\section{Referências}

1. Guillemin F, Bombardier C, Beaton D. Cross-cultural adaptation of health-related quality of life measures: literature review and proposed guidelines. J Clin Epidemiol 1993; 46(12):1417-1432.

2. Dantas RAS, Rossi LA, Costa MCS, Vila VSC. Qualidade de vida após revascularização do miocárdio: avaliação segundo duas perspectivas metodológicas. Acta Paul Enferm 2010; 23(2):163-168.

3. Minayo MCS, Hartz ZMA, Buss PM. Qualidade de vida e saúde: um debate necessário. Cien Saude Colet 2000; 5(1):7-18.

4. Góis CFL, Dantas RAS, Torrati FG. Qualidade de vida relacionada à saúde antes e seis meses após a revascularização do miocárdio. Rev Gaucha Enferm 2009; 30(4):700-707.

5. Novato TS, Grossi SAA, Kimura M. Instrumento de qualidade de vida para jovens com diabetes (IQVJD). Rev Gaucha Enferm 2007; 28(4):512-519.

6. Thompson DR, Yu CM. Quality of life in patients with coronary heart disease-I: Assessment tools. Health Qual Life Outcomes 2003; 1(1):42.

7. Leal A, Paiva C, Höfer S, Amado J, Gomes L, Oldridge $\mathrm{N}$. Evaluative and discriminative properties of the Portuguese Mac New Heart Disease Health-related Quality of Life questionnaire. Qual Life Res 2005; 14(10):23352341.

8. Aguiar CCT, Vieira APGF, Carvalho AF, MontenegroJunior RMM. Instrumentos de avaliação de qualidade de vida relacionada à saúde no Diabetes Melito. Arq Bras Endocrinol Metabol 2008; 52(6):931-939.

9. Beaton DE, Bombardier C, Guillemin F, Ferraz MB Guidelines for the Process of Cross Cultural Adaptation of Self-Report Measures. Spine 2000; 25(24):31863191.

10. Wild D, Grove A, Martin M, Eremenco S, McElroy S, Verjee-Lorenz A, Erikson P; ISPOR Task Force for Translation and Cultural Adaptation. Principles of Good Practice for the Translation and cultural Adaptation Process for Patient-Reported Outcomes (PRO) Measures: report of the ISPOR Task Force for Translation and Cultural Adaptation. Value Health 2005; 8(2):94-104.

11. Reichenheim ME, Moraes CL. Operacionalização de adaptação transcultural de instrumentos de aferição usados em epidemiologia. Rev Saude Publica 2007; 41(4):665-673.

12. Ferraz MB. Cross cultural adaptation of questionnaires: what is it and when should it be performed? J Rheumatol 1997; 24(11):2066-2068.

13. Herdman M, Fox-Rushby J, Badia X. A model of equivalence in the cultural adaptation of HRQoL instruments: the universalist approach. Qual Life Res 1998; 7(4):323-335.
14. Silva SA, Passos SRL, Carballo MT, Figueira M. Quality of life assessment after acutec oronary syndrome: systematic review. Arq Bras Cardiol 2011; 97(6):526-540.

15. Alcântara EC. Qualidade de vida após Infarto Agudo do Miocárdio: avaliação com os questionários Mac New QLMI e SF-36 [dissertação]. Uberlândia: Universidade Federal de Uberlândia; 2006.

16. Thompson AK, Juniper E, Meltzer EO. Quality of life in patients with allergic rhinitis. Ann Allergy Asthma Immunol 2000; 85(5):338-348.

17. Mendes EV. As situações das condições de saúde e os sistemas de atenção à saúde. In: Mendes EV. As redes de atenção à saúde. $2^{\mathrm{a}}$ ed. Brasília: Organização Pan-Americana da Saúde; 2011. p. 25-59.

18. Oldridge N, Guyiatt G, Crowe J, Feeny D, Jones N. Goal attainment in a randomized controlled trial of rehabilitation after myocardial infarction. J Cardiopulm Rehabil 1999; 19(1):29-34.

19. Thompson DR, Jenkinson C, Roebuck A, Lewin RJ, Boyle RM, Chandola T. Development and validation of a short measure of health status for individuals with acute myocardial infarction: the myocardial infarction dimensional assessment scale (MIDAS). Qual Life Res 2002; 11(6):535-543.

20. Alexandre NMC, Coluci MZO. Validade de conteúdo nos processos de construção e adaptação de instrumentos de medidas. Cien Saude Colet 2011; 16(7):30613068.

21. Tilden VP, Nelson CA, May BA. Use of qualitative methods to enhance content validity. J Nurs Res 1990; 39(3):172-175.

22. Thompson ND, Watson R. Mokken scaling of the Myocardial Infarction Dimensional Assessment Scale (MIDAS). J Eval Clin Pract 2011; 17(1):156-159.

Artigo apresentado em 08/08/2015

Aprovado em 27/05/2016

Versão final apresentada em 29/05/2016 
